\title{
Inhalt
}

\section{Abbildungs- und Tabellenverzeichnis $-\mathrm{XI}$}

\section{Konventionen -XIII}

1

1.1

1.2

1.3

2

2.1

2.1.1

2.1 .2

2.1 .3

2.2

3

3.2.1

3.2 .2

\section{Einführung - 1}

Verortung der Arbeit im linguistischen Feld -1

Untersuchungsgegenstand und -ziele - $\mathbf{4}$

Aufbau der Arbeit $\longrightarrow 7$

\section{Forschungsstand -12}

Marginal: Funktionswortarten in sprachhistorischen Grammatiken - 15

Nd. Sprachstufe des Untersuchungszeitraums: Mnd. - 15

Hd. Sprachstufen des Untersuchungszeitraums: Mhd. und Frnhd. - 18

In der Zusammenführung - 23

Polykategorial: Funktionswortarten in sprachhistorischen

Wörterbüchern - 24

Ausgewählte Fokussierungen in der sprachhistorischen

Funktionswortartforschung - 28

Textgrammatisch: Funktionswörter als Konnektoren - 29

Diachron: Sprachwandel bzw. Grammatikalisierung im Funktionswortbereich - 33

Eigene Vorarbeiten: Prototypengrammatische Perspektivierung — 37

Fazit: Desiderata und Perspektiven im Funktionswortbereich - $\mathbf{3 8}$

Theoretischer Rahmen: Kognitive und soziokulturelle

Dimension - 40

Kognitiv-funktionaler Grammatikbegriff — 42

Konstruktionsbasiertheit der Nichtflektierbaren - 48

Konstruktionsbegriff- 49

Konstruktionen kognitiv-grammatisch: Sprachliches

Konstruieren - 63

Konstruktionsbasierte Funktionswort-Kategorien: Profilierte

Relationen -69 
3.3 Funktionswortart-Konstruktionen als gradiente Prototypen-

Kategorien — 79

3.3.1 Zum Gradienz-Konzept - $\mathbf{8 1}$

3.3.2 Mehrdimensionale Prototypen-Kategorien - $\mathbf{8 8}$

3.4 Entwicklungen im Bereich der Grammem-Konstruktionen - 95

3.4.1 Entstehen und Wandel: constructionalization und constructional change- 98

3.4.2 Konstruktionale Grammatikalisierung als Prototypisierung - 103

3.4.3 Kategorienwechsel und Zunahme an Polykategorialität - 116

3.5 Konstruktionen in soziokultureller Hinsicht -123

3.5.1 Desiderat: Social Cognitive Linguistics - 126

3.5.2 Sprache als soziale Gestalt nach Feilke - 131

3.5.3 Kulturanalyse: Konstruktionen in Praktiken - 146

3.5.4 Weiterführend: Praktik und Text(-Konstruktion) -160

3.6 Zusammengefasst: Das kognitiv-funktionale Theoriegerüst - 167

4 Literater Sprachausbau: Historische Rechtsschriftlichkeit - 171

4.1 Lebensweltliche und sprachhistorische Einbettung - 173

4.1.1 Die Stadt im Spätmittelalter und der Frühen Neuzeit: konstitutives Recht - aufkommende Kanzleien - wachsende Schriftlichkeit - 175

4.1.2 Städtische Kommunikation: Historische Schriftlichkeit im Fokus - 179

4.1.3 Exkurs: Zum Verständnis von Schriftlichkeitskonzepten -187

4.2 Rechtsschriftlichkeit im 13. bis 16. Jh. - 211

4.2.1 Überblick: Historische Rechtstexte/-schriftlichkeit - 212

4.2.2 Schriftlichkeitsforschung: Recht, Literalität, Textualisierung - $\mathbf{2 1 7}$

4.2.3 Kanzleisprachenforschung: Syntaktischer Fokus - 225

4.3 Literater Sprachausbau: Sprachliche Komplexität - 231

4.3.1 Überblick: Sprachausbauforschung - 232

4.3.2 Literater Sprachausbau nach Maas - 241

4.3.3 Literate Konstruktionen und sprachliche Komplexität - 261

4.3.4 Funktionswort-Konstruktionen im literaten Sprachausbau: Historisches Recht-Schreiben - 270

4.4 Zwischenbilanz: Sprachausbaukonstruktionen in der historischen Rechtsdomäne - 274 
$5 \quad$ Korpusgrundlage und Methodik -279

5.1 Überblick: Korpuslinguistisches Vorgehen - 279

5.2 Untersuchungsgrundlage: Auswahl der mnd. Rechtstexte $2 \mathbf{2 8 6}$

5.3 Methodik 289

5.3.1 Konstruktionen im Korpus - 291

5.3.2 Kulturanalytische Perspektivierung — 296

$6 \quad$ Untersuchungsergebnisse -299

6.1 Sprachausbaubereich I: Subordinative (Schnittstellen)Phänomene-299

6.1.1 Space builder-Konstruktionen - 304

6.1.2 Modifizierende Funktionswort-Konstruktionen - 312

6.2 Sprachausbaubereich II: Informationsdichtes construal - 414

6.2.1 Präpositional-konstruktionales Modifizieren mit deverbalen Nomen - 417

6.2.2 Präpositionale Konstrukte als Registereinträge und Überschriften: van-Konstruktion(en) $-\mathbf{4 4 3}$

6.3 Sprachausbaubereich III: Textorganisation - Text-

Konstruktionen - $\mathbf{4 5 1}$

6.3.1 Adverbielle und konjunktionale textgliedernde Konstruktionen - $\mathbf{4 5 5}$

6.3.2 Kanzleisprachentypisch: Zur Item-Konstruktion und ihren Konkurrenten - 471

6.3.3 Thema-Konstruktion: were yt sake dat-Konstruktion und Ähnliches 479

6.4 Zusammenfassung $-\mathbf{4 9 5}$

7 Im kulturanalytischen Horizont: Zu den herausgearbeiteten Konstruktionen - $\mathbf{5 0 2}$

7.1 Interpretationsrahmen: Institutionalisierung von Recht und RechtSchreiben - $\mathbf{5 0 3}$

7.2 Global gedacht: Phänomene der Textualisierung (Lesetexte) - $\mathbf{5 0 7}$

7.3 Zur Praktikentypik ausgewählter Konstruktionen: TexttypenVergleich - $\mathbf{5 1 2}$

7.3.1 Konditionales Relationieren - $\mathbf{5 1 5}$

7.3.2 Verdichtungsbedarf: Deverbales Konstruieren - $\mathbf{5 2 2}$

7.4 Im Ergebnis - $\mathbf{5 2 7}$ 
$\mathbf{X}$ - Inhalt

8 Zusammenfassung und Weiterführendes - 529

8.1 In prägnanter Kürze: Zusammenfassung der Ergebnisse - 529

8.2 Reflexion der eingenommenen Perspektive - $\mathbf{5 3 2}$

8.2.1 Funktionswort-Konstruktionen im Sprachausbau - $\mathbf{5 3 2}$

8.2.2 Linguistik und soziologische Praxistheorie - $\mathbf{5 3 4}$

8.3 Weiterführende Frage: Sprachrückbau des

Niederdeutschen? $\mathbf{5 3 6}$

Literatur -539

Sachregister -597 